\title{
Evidence for avian cell autonomous sex identity (CASI) and implications for the sex-determination process?
}

\author{
M. Clinton • D. Zhao $\cdot$ S. Nandi • D. McBride
}

Published online: 29 November 2011

(C) Springer Science+Business Media B.V. 2011

\begin{abstract}
For the majority of animals, males and females are obviously different in terms of appearance, behaviour and physiology, and until recently, these differences were considered to be the result of hormone actions. However, there is now considerable evidence that the development of some sexually dimorphic structures/behaviours is a function of properties inherent to male and female cells (hormone independent). The relative contribution of hormones and cellular identity to the development of the phenotype is not clear and is likely to vary from species to species. The study of gynandromorph birds and chimeric embryos has greatly assisted efforts to distinguish between the effects of hormones and inherent cellular factors on phenotype. It is now clear that in birds, male/female differences are not primarily the result of hormone action and that male and female somatic cells possess a cell autonomous sex identity (CASI). Here, we review evidence for CASI in birds and discuss the implications for the process of sex determination.
\end{abstract}

Responsible Editors: Tariq Ezaz and Jennifer Graves.

M. Clinton $(\bowtie) \cdot$ D. Zhao $\cdot$ S. Nandi $\cdot$ D. McBride

Department of Developmental Biology,

The Roslin Institute and Royal (Dick) School of Veterinary

Studies, University of Edinburgh,

Easter Bush,

Midlothian, UK EH25 9RG

e-mail: Michael.clinton@ed.ac.uk

URL: www.roslin.ed.ac.uk
Keywords CASI · gynandromorph · dosage · avian · chicken $\cdot \mathrm{Z}$ chromosome $\cdot \mathrm{W}$ chromosome $\cdot$ gonad . dimorphic $\cdot \operatorname{sex} \cdot$ sexual $\cdot$ chimeras
Abbreviations
GFP
Green fluorescent protein
CASI
Cell autonomous sex identity
$\mathrm{AMH}$
Anti-Mullerian hormone
AROM Aromatase
Sry
Sex determination region Y-chromosome gene
DMY The DM-domain gene on the Y chromosome
SOX9 Sry-box containing gene 9
DMRT1 Doublesex and Mab-3-related transcription factor 1
$\mathrm{Mb} \quad$ Mega base pairs
$\mathrm{Kb} \quad$ Kilo base pairs
MHM Male hypermethylated region

\section{Introduction}

Previously, the general view of vertebrate sex determination equated gonadal differentiation with sexual development: it was thought that the action of a 'master regulator' gene located on a sex chromosome determines whether the genital ridge develops as a testis or ovary, and then the male or female phenotype of other somatic tissues is imposed by gonadal 
hormones (regardless of genotype). However, it is now widely accepted that hormone-independent characteristics that are inherent to male and female cells, also play a role in determining the final phenotype (reviewed in Arnold 2003; Davies and Wilkinson 2006; Blecher and Erickson 2007; Arnold and Chen 2009; Ngun, et al. 2011). This raises the question about the extent of the contribution that these cell autonomous properties make to structural and functional differences between males and females, and the answer seems to be that the effect varies from species to species.

In mammals, hormones are still considered to play a dominant, but not exclusive, role in establishing the phenotype of non-gonadal tissues, while there is growing evidence that the inherent sex identity of individual male and female cells plays a much more significant role in defining sexual dimorphisms in birds, and possibly other vertebrates. Here, we will review the evidence for cell autonomous sex identity (CASI) and consider both the molecular basis of CASI and the possible implications of CASI for sex determination in birds.

\section{Evidence for CASI}

For some time, it has been clear that the development of sex differences in the avian brain are not solely due to sex differences in gonadal hormone secretion (reviewed in Arnold 1997). Recent studies of gynandromorph birds have demonstrated that this is also true for other somatic tissues and have suggested that this CASI of individual cells plays a major role in defining the phenotype of non-gonadal tissues (Agate et al. 2003; Zhao et al. 2010).

Gynandromorphs are naturally occurring birds that display a striking bilateral asymmetry: one side of the bird appears to be male while the other side appears to be female (gyne=female; andro=male; $\operatorname{morph}=$ form). For example, in the gynandromorph chicken shown here (Fig. 1), the right side of the bird is femalecoloured (brown) and has a slight build with a small wattle and no obvious spur. In contrast, the left side is white, has a heavy breast musculature and bone structure, and has a large wattle and obvious spur; all characteristics typical of a male bird. For gynandromorph birds, this asymmetry has only been
Fig. 1 Gynandromorph chicken. a Photograph of gynandromorph. Right side of bird appears female with brown plumage, small wattle and slight build while left side of bird displays features typical of a male: white plumage, large wattle, large breast musculature and spur. b Schematic illustrating distribution of female (ZW) and male (ZZ) cells. Tissues on the right side are composed predominately of female cells and tissues on the left side are composed predominately of male cells

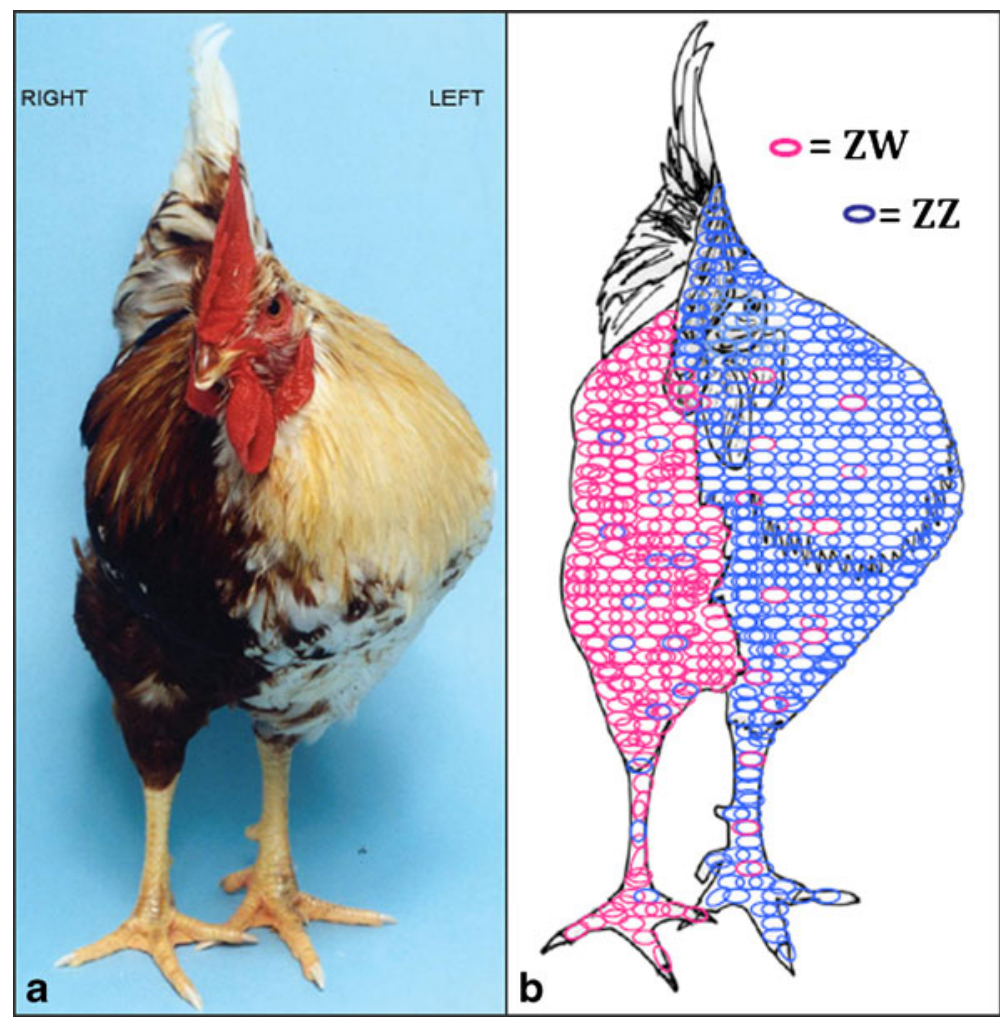


reported to occur about the anterior:posterior/dorsal: ventral midline, and although previous reports had suggested that only male-left:female-right gynandromorphs were viable, it is now clear that male-right: female-left birds also occur. In a gynandromorph zebrafinch study, Arnold and colleagues confirmed earlier reports that asymmetry was not simply superficial and showed that the neural song circuit on the right side of the brain had a more masculine phenotype than that on the left (Cock 1955; Agate et al. 2003). In a more recent study (Zhao et al. 2010), measurements performed on individual tissues demonstrated that this asymmetry applied to all paired tissues throughout the animal; tissues on the male side were larger and heavier and bones were longer and denser than those from the female side.

A long-standing theory on the etiology of gynandromorphs proposed that these birds arose because of the loss of a single sex-chromosome on one side of the animal at the two-cell stage of development (Cock 1955). The gynandromorph zebrafinch analysis, although not establishing a formal karyotype, suggested that this was not the case (Agate et al. 2003), and the study by Zhao et al. (2010) has now established, unequivocally, that these birds are not anueploids: tissues are composed of normal diploid male and female cells. A thorough analysis of the cellular composition of multiple tissues established that the majority of cells on the side that appears female contain one $\mathrm{Z}$ and one $\mathrm{W}$ sex chromosome, while the majority of cells on the side that appears male contain two $\mathrm{Z}$ sex chromosomes: these analyses have demonstrated that gynandromorph birds are undoubtedly male:female chimeras. The presence of both ZZ- and ZW-containing cells suggests that it is highly unlikely that these birds arise as a consequence of mutation at the two-cell stage of development and would support the hypothesis that gynandromorphs arise as a result of failure of extrusion of a polar body during meiosis and subsequent fertilisation of both a Z- and W-bearing female pronucleus (Hollander 1975).

The demonstration that gynandromorphs are male/ female chimeras and that the phenotype of nongonadal tissues reflects cellular genotype rather than circulating hormones is at odds with the classical view of vertebrate sex determination. These results strongly suggest that the avian phenotype is dependent on the nature of the cells comprising the individual tissue rather than being imposed by the type of gonad formed: both sides of these animals are exposed to an identical profile of gonadal products yet each side responds differently to these stimuli (Agate et al. 2003; Zhao et al. 2010). These analyses led to the conclusion that male and female chicken somatic cells possess a cell-autonomous sex identity (CASI). An analysis of the gonads present in the three gynandromorph chickens studied also supports the concept of CASI. Of the three birds, one carried a testis-like gonad on the left side, a second carried an ovary-like gonad on the left side, and the third carried an ovo-testis on the left side. Detailed analyses demonstrated that the morphological appearance of the gonads conformed to the cellular composition in that the structures which appeared to be testis or ovary were composed principally of ZZ- or ZW-containing cells, respectively, while the 'ovo-testis' comprised a mixture of ZZ- and ZW-containing cells.

To test the concept of CASI, a parallel transplantation study was performed with the objective of generating chick embryos with male/female chimeric gonads. This study generated embryos with gonads composed of different mixtures of male and female cells and examined both same-sex (male donor/male host \& female donor/female host) and mixed-sex chimeras (male donor/female host and female donor/ male host). Chimeras were generated by transplantation of sections of presumptive mesoderm from the left side of green fluorescent protein (GFP)-expressing donor embryos (McGrew et al. 2004) at developmental stage 12 (day 2) (Hamburger and Hamilton 1951) to replace the equivalent tissue of wild-type host embryos at the same stage of development (Fig. 2). Eggs containing the transplanted embryos were then sealed and re-incubated for a further 7 days by which time the embryo had developed to $\mathrm{H} \& \mathrm{H}$ Stage 35. By this point in development, the transplanted GFP cells had multiplied, migrated to different locations on the left side of the developing embryo, and differentiated to contribute to various tissues, including the gonad. At H\&H Stage 35, the normal embryonic male gonad is composed of a thin layer of cortex tissue surrounding a medullary region which contains the developing sex cords (expressing anti-Mullerian hormone-AMH) separated by interstitial connective tissue. In contrast, the female left ovary comprises a greatly thickened cortex surrounding a smaller less-structured medullary region (expressing aromatase). In all same-sex chimeras 


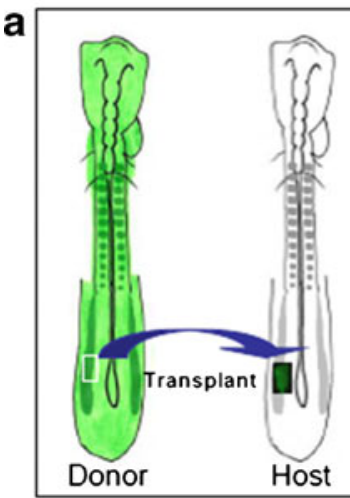

Embryos at day 2 of development

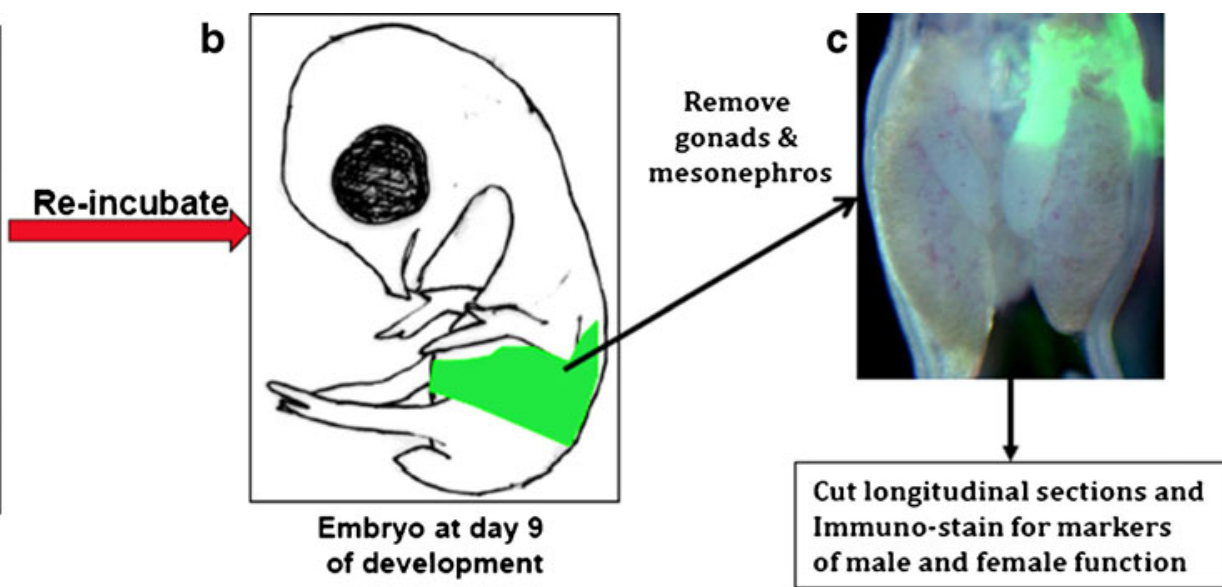

Fig. 2 Schematic illustrating transplantation procedure used to generate chimeric chick embryos. a Section of lateral-plate mesoderm is removed from left side of GFP-expressing embryo and transplanted to equivalent region of wild-type embryo of same or opposite sex. Manipulations are performed only on the left side of embryos as the sex of the donor embryo is unknown at the time of transplantation, and only the left gonad develops

generated, the male or female donor cells were integrated into all somatic compartments of the respective host testis or ovary (cortex, sex cords and interstitial tissue) (Fig. 3a). Moreover, when donor cells integrated into the appropriate 'functional' compartment of the host gonad, donor male cells expressed AMH and donor female cells expressed aromatase. The results from the same-sex chimeras demonstrate that donor cells placed in a host embryo are able to correctly interpret and respond to developmental signals to multiply, migrate and differentiate appropriately. Cells that migrate into the embryonic region that will form the gonad are able to respond to the relevant signals and differentiate into any gonadal cell type. In a complete contrast, in mixed-sex chimeras, donor cells appear to be incapable of contributing to specialised compartments of the host gonad. Female donor cells in the testis of a male host cannot be recruited into the functionally 'male' Sertoli cell compartment, and male donor cells in the ovary of a female host are excluded from the functionally 'female' compartment. This effect is most obvious in the more structured testis: in female donor/male host chimeras, donor female cells are clearly confined to the interstitial tissue between the developing sex cords (Fig. 3b), and under these circumstances, do not express either the host or donor markers. The failure of donor cells to integrate into the specialised in females. Eggs are re-incubated for 7 days. b By H\&H Stage 35 , transplanted GFP-expressing cells have contributed to the majority of tissues in the region between fore- and hind-limb on the left side. c Photograph of gonads and mesonephros following dissection. Green colour demonstrates location of GFP-expressing cells. Tissues are sectioned and processed for immuno-histochemistry

structures of the host gonad in chicken mixed-sex chimeras is at odds with the findings from mammalian mixed-sex chimeras, where donor cells contributed to all compartments of the host gonad (Patek et al. 1991; Burgoyne et al. 1988). These findings not only support the concept that chicken somatic cells possess a CASI but also suggest that under normal developmental influences, these cells, unlike mammalian cells, cannot be induced to acquire the functional identity of the opposite sex. Even more surprisingly, it transpires that not only do donor cells fail to acquire a functional host identity, in mixed-sex chimeras, donor cells can actually retain a functional donor identity. This is illustrated in Fig. 4, which shows sections through a chimeric gonad derived from donor male cells and host female cells. In this instance, the donor male cells have contributed the majority of the cells to the anterior portion of the host 'ovary'. These sections have been stained for GFP to identify donor cells and for the male and female 'functional' markers, AMH and aromatase. Again, it is clear that there is no co-localisation of the donor GFP and the functional marker of the host, aromatase. However, when immuno-stained for the functional marker of the donor, it is obvious that the donor cells express high levels of AMH. In addition, not only do the donor cells express $\mathrm{AMH}$, it is clear that the region containing mostly donor cells is being 


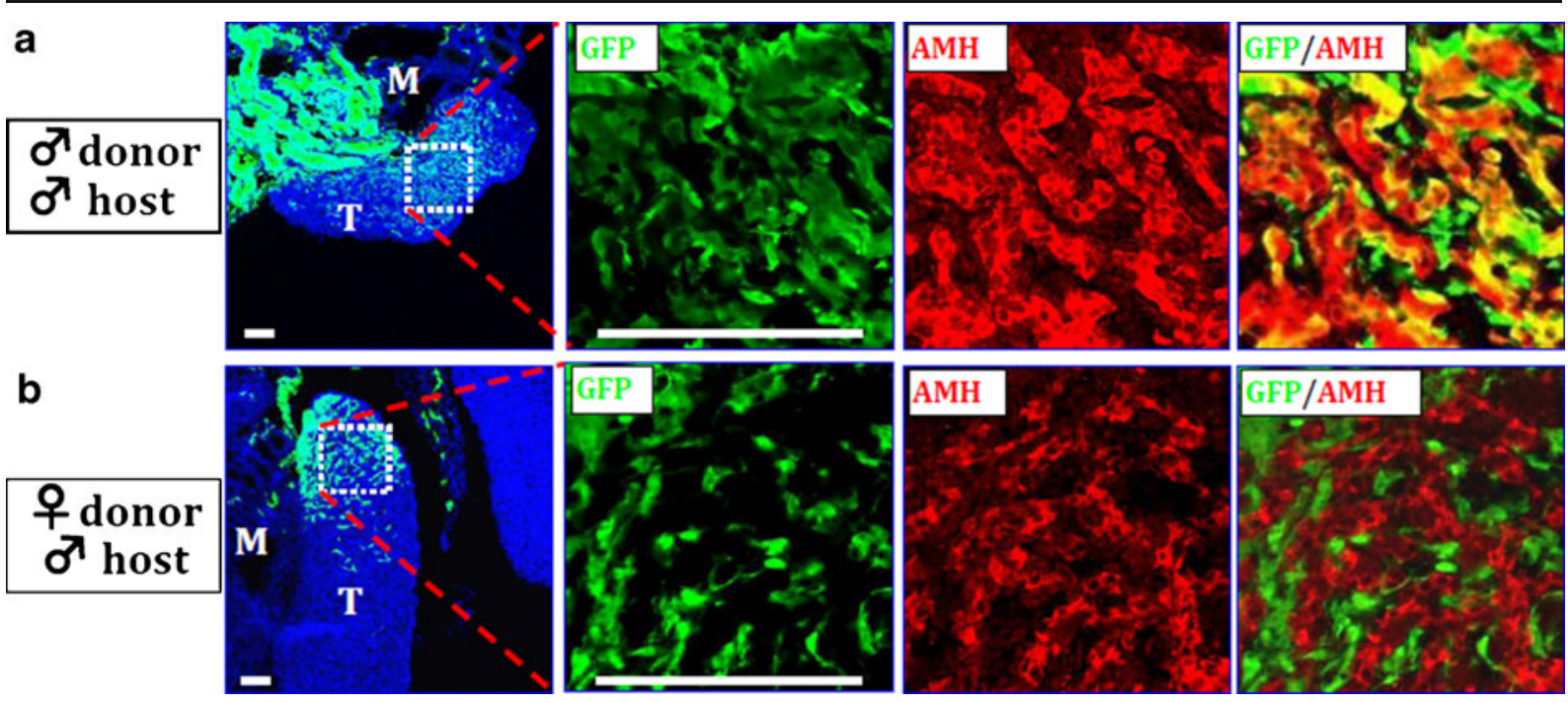

Fig. 3 Distribution and developmental fate of donor cells in host gonads of chimeric chicken embryos. a Same-sex chimera. Histological section through chimeric gonad composed of male donor GFP-expressing cells and male wild-type host cells. Left panel shows low-power image of section through mesonephros (M) and testis (T). Remaining panels show higher-power images of region indicated in left panel. Sections were immuno-stained for expression of GFP (green fluorescent protein) and $\mathrm{AMH}$ (anti-Mullerian hormone). Right panel shows overlay of GFP and AMH images and demonstrates that donor-GFP cells can express AMH and can contribute to developing sex cords and interstitial tissue. b Mixed-sex

remodelled into the characteristic sex cord structures typical of a developing testis. It seems that transplanted cells can respond to developmental signals to form the appropriate tissue (a gonad), but the sexual phenotype of that tissue depends on the sexual identity (CASI) of the cells involved. The donor and host cells shown in Fig. 4 are clearly subject to identical developmental signals, yet cells of different genotypes respond differently - the female cells generate an ovary and the male cells generate a testis (compare AROM and AMH panels). This clearly demonstrates that chicken somatic cells possess a cell autonomous sex identity and that, under normal developmental conditions, this CASI determines whether an ovary or a testis is formed.

This raises the question of what exactly defines the sexual phenotype: during normal development, it is clear that both CASI and hormones influence the final tissue phenotype, but which, if either, is the major factor? Data from mammalian studies would suggest that hormones undoubtedly play the dominant role in chimera. Histological section through chimeric gonad composed of female donor GFP-expressing cells and male wildtype host cells. Left panel shows low-power image of section through mesonephros (M) and testis (T). Remaining panels show higher-power images of region indicated in the left panel. Sections were immuno-stained for expression of GFP and AMH. Right panel shows overlay of GFP and AMH images and clearly demonstrates that female donor-GFP cells do not express AMH and do not contribute to developing sex cords. Female donor cells are restricted to the interstitial tissue and excluded from functional structures of host testis in mixed-sex chimeras (white bars represent $100 \mu$ )

determining phenotype, but it may be that assumptions based on mammalian findings have led to a bias in interpreting data from other species. For example, the avian wattle has long been described as a hormone-sensitive tissue because wattle size in newly hatched chicks can be increased to proportions normally found in sexually mature males by administration of testosterone, and conversely, castration of mature males results in a pronounced reduction in wattle size (Barnard 1983; Fennell and Scanes 1992; Briganti et al. 1999). However, experiments/procedures of this nature are performed on individual, different birds where it is very difficult to calculate the contribution of CASI. A different picture appears when considering the wattles in a gynandromorph bird where the effects of hormones and CASI can be compared within a single individual. In the example shown here (Fig. 5), the wattle on the male side is 2.4 times larger by weight than the wattle on the female side. This particular bird carried an apparently normal testis complete with sperm, and although the exact 


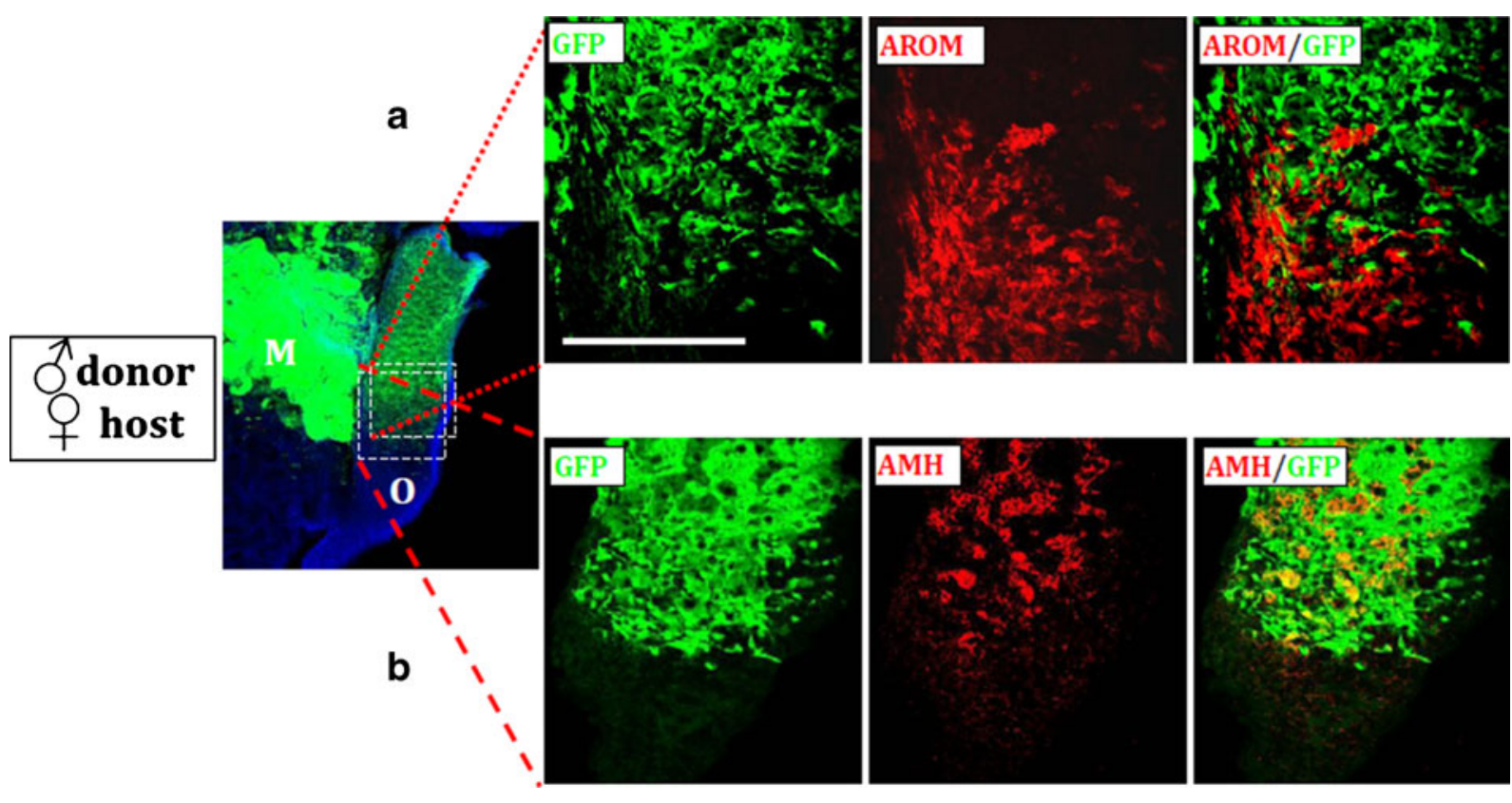

Fig. 4 Distribution and developmental fate of cells in gonad of mixed-sex chimera with high donor contribution. Histological sections through chimeric gonad composed of male donor GFPexpressing cells and female wild-type host cells. Left panel shows low-power image of section through mesonephros $(M)$ and ovary $(O)$. a Higher-power images of region indicated in left panel. Sections were immuno-stained for expression of GFP and AROM (aromatase). Right panel shows overlay of GFP and AROM images and demonstrates that donor-GFP

composition of circulating gonadal hormones is unknown, the hormonal environment for both wattles is clearly identical, and therefore, cannot account for the difference in size of right and left wattles. The only known difference between these tissues is that one wattle is composed predominately of male cells and the other is composed predominately of female cells. In a second of the gynandromorph chickens studied, the male wattle was 4.1 times larger than the female wattle, and in this bird, the gonad developed as an ovary (Zhao et al. 2010). Hormones undoubtedly affect the size of the male or female wattle in gynandromorph birds, but our observations would suggest that the CASI of the cells comprising these tissues is the major factor in determining size.

In general, it seems obvious that the overall phenotype must be dependent on both hormones and CASI, but from the evidence obtained from these gynandromorph studies, it would seem that CASI plays a more significant role in determining phenotype in birds than in mammals. On the basis that birds cells do not express aromatase and do not co-localise with functional structures of the host gonad. b Higher-power images of indicated region of neighbouring section. Sections were immuno-stained for expression of GFP and AMH. Right panel shows overlay of GFP and AMH images and demonstrates that male donor cells in a host ovary express the male marker $\mathrm{AMH}$ and generate male-specific sex cords (white bar represents $100 \mu$ )

have an effective CASI, we have proposed a novel model of the process of sex-determination in birds (Fig. 6).

A second question posed by these findings is whether the development of an active CASI with such a marked effect on phenotype is specific to birds or is a more widespread feature in sexual development generally. The physical manifestation of an effective CASI and the phenomenon of gynandromorphism are, of course, not limited to birds with well-known examples in insects and crustacean (Spencer 1927; Chace and Moore 1959; Bowen and Hanson 1962; Johnson and Otto 1981; Morgan 1991) (Fig. 7). In insects, it is well documented that somatic cells have an inherent sex identity (Gilbert 2006; Wolpert et al. 2006); however, little is known about the mechanism of sex determination in crustaceans (Staelens et al. 2008). This may suggest that an effective CASI must be limited to species where gynandromorphs have been identified, but we would caution against such a superficial 


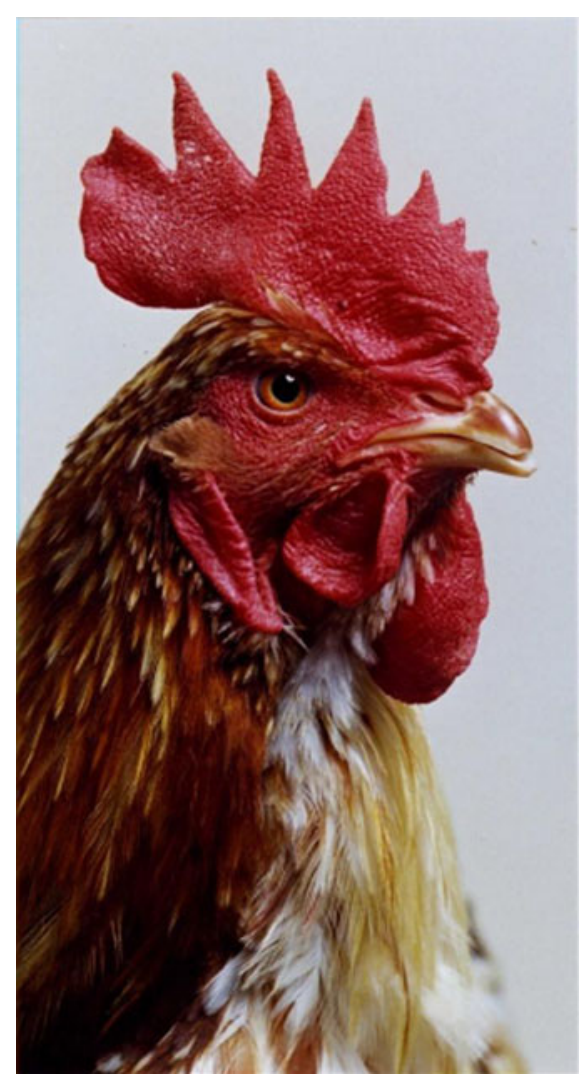

Fig. 5 Influence of CASI on development of hormonesensitive tissue. Photograph of gynandromorph chicken showing wattles on female (right) and male (left) side of bird. It is clear from the image that the wattle on the male side is significantly larger than that on the female side

conclusion for the simple reason that unless the males and females of a species are markedly different in appearance or size, gynandromorph animals may be very difficult to identify. Indeed, there is good evidence for an effective CASI in a marsupial mammal, the wallaby, where the formation of the mammary gland and scrotum is independent of gonadal hormones (O et al. 1988; Graves 1996).

\section{Molecular basis of CASI}

As regards the molecular basis underlying male and female CASI, clearly the most obvious difference between male and female somatic cells is the sex chromosomes. In birds, of course, the sex chromosomes are designated $\mathrm{Z}$ and $\mathrm{W}$, with the females the heteromorphic sex (ZW) and the males the homomorphic sex
(ZZ). Although the $\mathrm{Z}$ and $\mathrm{W}$ chromosomes share a common ancestry, a lack of meiotic pairing along most of the length of the female-specific $\mathrm{W}$ chromosome has resulted in its degeneration in both size and sequence content (Marshall Graves and Shetty 2001). The chicken $\mathrm{Z}$ chromosome is around $85 \mathrm{Mb}$ long and encodes approximately 1,000 genes (Bellott et al. 2010), while the small and highly heterochromatic W chromosome is poorly represented with only $0.26 \mathrm{Mb}$ of sequence available and only a handful of possible protein-coding genes identified (http://www.ensembl. org/index.html).

In species with heteromorphic sex chromosomes, it is thought that as genes are lost from the degenerating sex chromosome, a genetic regulatory mechanism must evolve to equalise the phenotypic expression of characteristics determined by genes on the $\mathrm{Z}$ chromosome. The shorthand description of this mechanism is 'dosage compensation', and this has been accomplished by a variety of different mechanisms in different species. These include, elevating X-chromosome-linked gene expression in male drosophila, lowering X-chromosomelinked gene expression in female Caenorhabditis elegans, and the random inactivation of one $\mathrm{X}$ chromosome in mammals (reviewed in Zarkower 2001; Straub and Becker 2007).

The differences between male and female cells that underlie CASI are obviously a function of the sex chromosomes, and we believe that the sex identity evident in avian male and female somatic tissues is a result of the type of dosage compensation system utilised in birds.

Surprisingly, until relatively recently, it was widely accepted that avian $\mathrm{Z}$ chromosomes were not subjected to the process of 'dosage compensation'. This is surprising because the consequence of no dosage compensation would be that all Z-chromosome genes would be expressed at relatively higher levels in males than in females. This belief was mainly based on limited evidence regarding sex differences in the activity of the Z-linked aconitase enzyme (Baverstock et al. 1982). However, in 2001, this view was overturned when we established that the levels of expression of some $\mathrm{Z}$ chromosome genes were equalised in male and female birds, suggesting some form of dosage compensation (McQueen et al. 2001). This study examined transcript levels of only nine $\mathrm{Z}$ chromosome genes in male and female chick embryos 


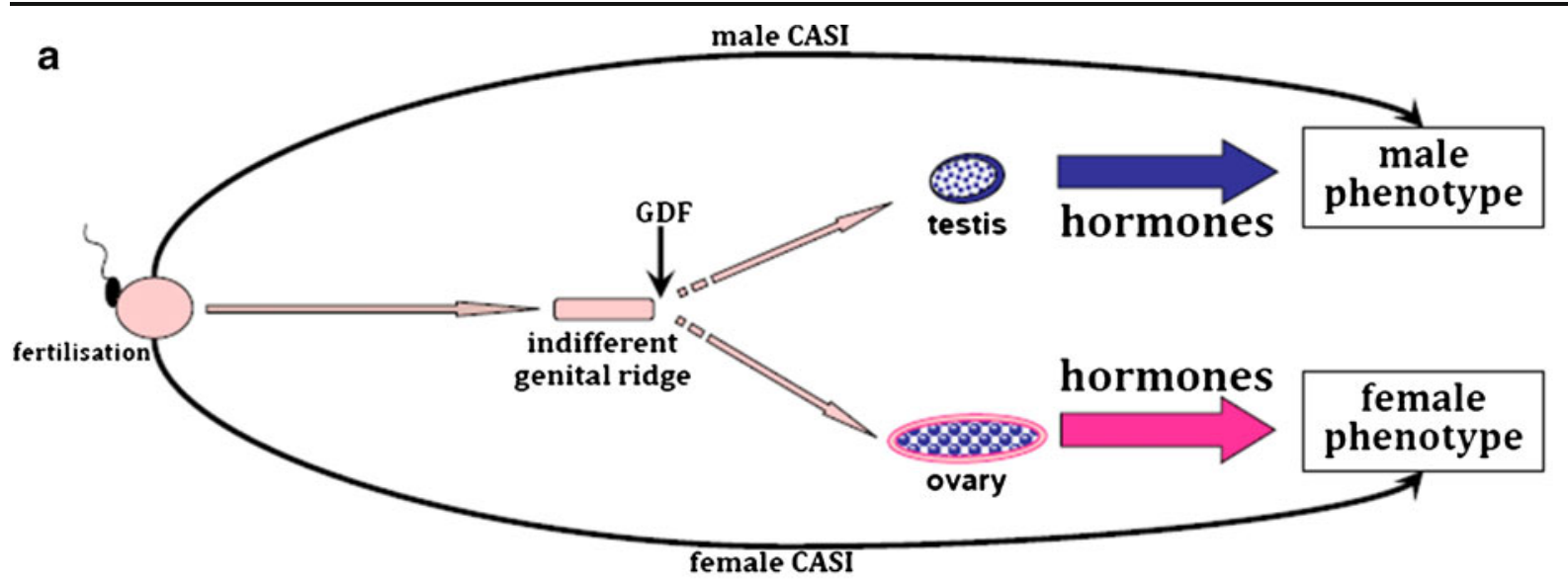

-Phenotype largely dependent on hormones

b

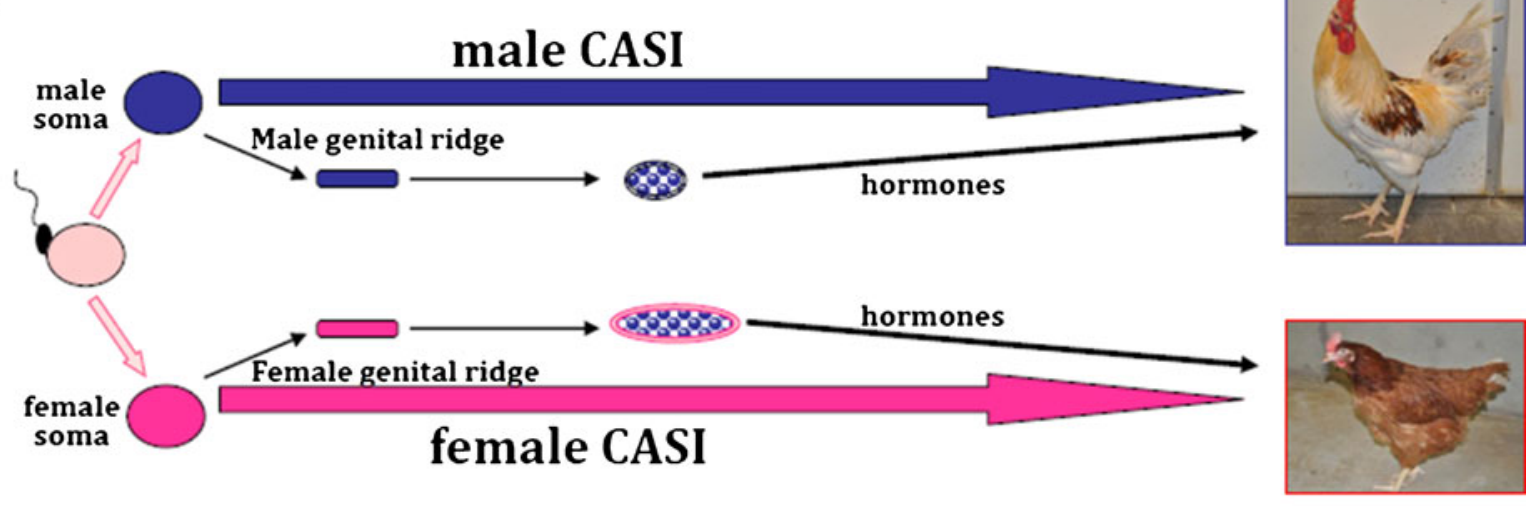

\section{-Phenotype largely dependent on CASI}

Fig. 6 Schematic illustrating relative contribution of hormones and CASI to development of the sexual phenotype. a Standard model of vertebrate sex determination where effect of hormones outweighs the effect of CASI in establishing sexually dimorphic aspects of non-gonadal tissues. b Proposed model of avian

prior to the development of the gonads (very few genes had been mapped to the $\mathrm{Z}$ chromosome by 2001). Although we observed very heterogeneous levels of expression between individuals of the same sex, similar averaged levels of expression were found in males and in females for six of these genes. This led to the conclusion that these genes must be subject to some form of dosage compensation but that this regulation did not affect all $\mathrm{Z}$ chromosome genes. These studies established for the first time that a significant proportion of avian $\mathrm{Z}$ chromosome genes were likely to be subject to dosage compensation but gave no real indication as to the extent of this sex determination where CASI plays a major role in determining male:female differences in appearance, behaviour and physiology. In both models, CASI is established at fertilisation (GDF gonad differentiation factor)

compensation or to the nature of the mechanism involved. Since then, a large number of studies have been carried out that have extended and confirmed our initial findings, in that the majority of $\mathrm{Z}$ chromosome genes were examined and a significant proportion shown to be compensated. It is also clear from these studies that a large number of genes across the avian $\mathrm{Z}$ chromosome are expressed at a higher level from the two male $Z$ chromosomes than from the single female $\mathrm{Z}$ chromosome (e.g., Itoh et al. 2007; Ellegren et al. 2007; reviewed in McQueen and Clinton 2009). This strongly argues against a global all-inclusive system of dosage compensation across 
Fig. 7 Examples of arthropod bilateral gynandro morphs. a Rosy gypsy moth, female on the right hand side, male on the left hand side. (Photograph by USDA APHIS PPQ

Archive, USDA APHIS PPQ, http://www.bugwood. org). b Lobster, male on the right hand side, female on the left hand side (picture taken from review by Aw and Levin 2008, originally appeared in Bangor Daily News)
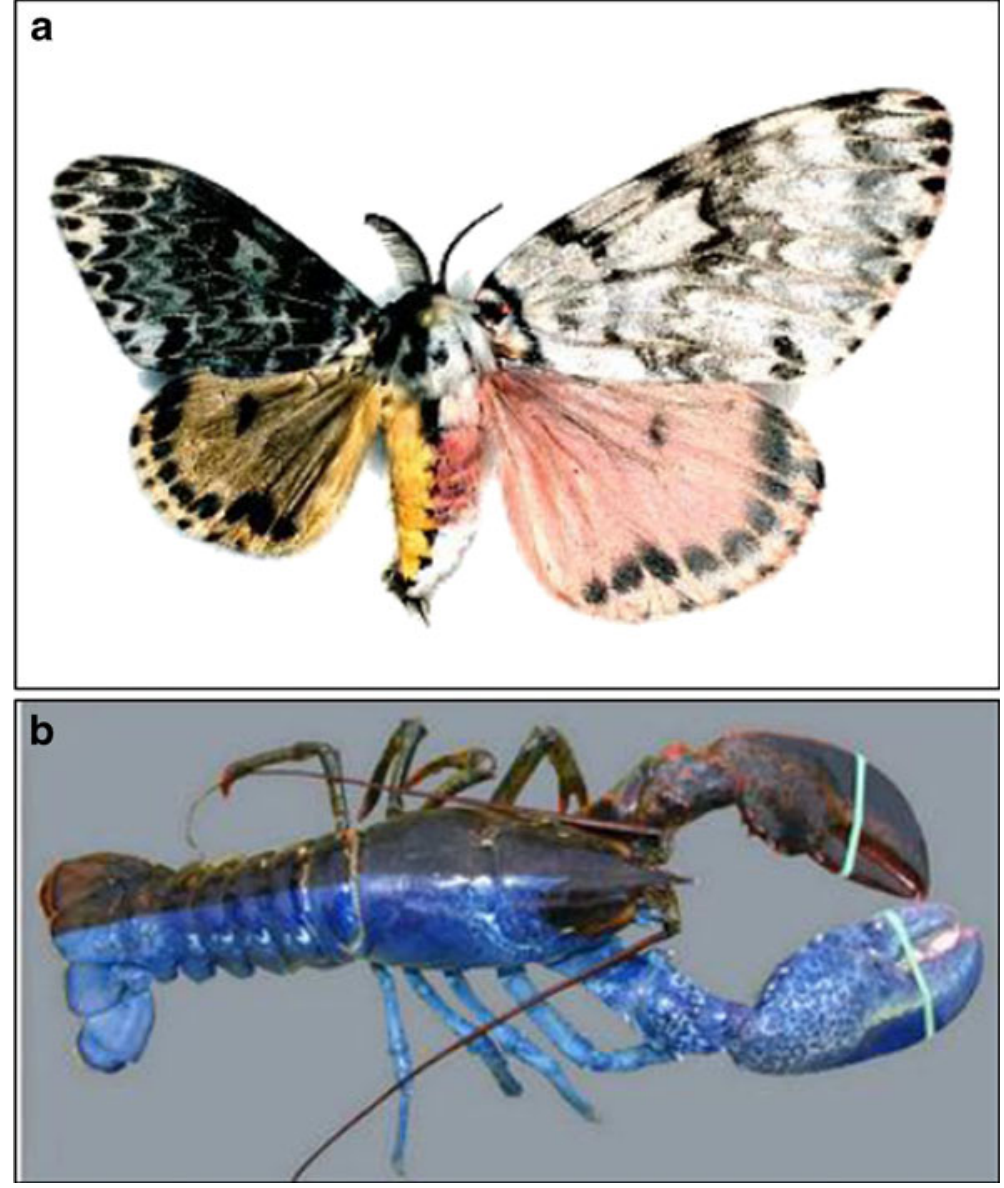

the avian $\mathrm{Z}$ chromosome and suggests that many genes are not compensated or only partially compensated.

\section{CASI genes}

We believe that the male and female cellular identity evident in avian tissues is a direct result of a large number of sex chromosome genes being expressed at different levels in male and female cells. These CASI genes will include $\mathrm{W}$-chromosome specific genes and non-compensated $\mathrm{Z}$ chromosome genes. We predict that CASI genes will be expressed in a sexually dimorphic fashion in all cells/tissues at all stages of development (including adult tissues).

While published studies would appear to support the existence of a large number of CASI genes, the majority of these male:female comparisons were performed on tissues from either adult birds or from embryos at stages after the development of the gonads, making it impossible to determine whether the sexually dimorphic expression is due to a lack of dosage compensation or a result of hormone action. However, a small number of studies have been carried out on tissues from stages before gonadal differentiation, and these strongly support the concept of CASI genes. Most notably, the study performed by Zhang et al. (2010) identified over 200 sexually dimorphic transcripts in H\&H Stage 4 male and female embryos (Scholz et al. 2006; Lee et al. 2009; Zhang et al. 2010). On the basis of these and other studies (Melamed and Arnold 2007; Itoh et al. 2007; Mank and Ellegren 2009; McQueen and Clinton 2009), we believe that as many as 300 CASI genes exist.

At this stage, it is not clear whether CASI is likely to be manifested by the actions of a small number of genes with significant effects or by the cumulative activity of a large number of genes with small effects - or indeed, both. 


\section{Implication for sex determination}

Sex determination is often equated solely with gonadal differentiation but is actually a life-long process. For species with genetic sex determination, sex determination per se obviously occurs at fertilisation when one sex acquires a pair of homomorphic sex chromosomes and the other, a pair of heteromorphic sex chromosomes. Gonadal development occurs later in development when the action of a sex chromosome gene(s) determines whether the genital ridge differentiates into a testis or an ovary, and, finally, hormones produced by the gonad then affect the phenotype of non-gonadal tissues.

Here, we review current understanding of sexdetermination in birds and consider how an effective CASI may influence the development of the gonads and the sexual phenotype.

Sex-determining mechanisms and gonad-determining genes

During gonadal development, a specific trigger is thought to initiate a hierarchical cascade of regulatory gene activity in the indifferent genital ridge that induces the development of testes in males and ovaries in females. Vertebrate gonads are highly conserved structures, so it is not surprising that the genes regulating the morphological differentiation of the gonads appears to be largely conserved across different vertebrate species (Morrish and Sinclair 2002; Ferguson-Smith 2007; Marshall Graves and Peichel 2010; Chue and Smith 2011). However, the 'trigger' that initiates testis or ovary development is clearly not conserved. In mammals, this trigger is a localised, short burst of expression of a gene encoded on the male-specific Y chromosome (Sekido and Lovell-Badge 2009; Kashimada and Koopman 2010). This gene, Sry, is expressed in the male genital ridge for a short period immediately before the first appearance of male/female differences. Sry represented a 'master regulator' gene that was originally thought to divert the developing gonad onto the path of testis differentiation and off the 'default' ovarian path. Although the discovery of Sry represented a major breakthrough, in many ways, it also heralded a false dawn: attempts to identify 'sex-determining genes' in other species focused on matching the Srytemplate, and this may not always have been appropriate. Despite major efforts in many species, only one other example quite like Sry has been identified: this gene is known as DMY, it is encoded on the Y chromosome and its expression can initiate testis development in either genetically male or female fish (Matsuda et al. 2007). However, DMY appears only in one fish species, Medaka, and may have only recently evolved (Kondo, et al. 2003).

Although the mammalian model is seen as a template for all vertebrate sex-determination systems, it may well be that some vertebrate gonaddetermining genes conform to a mechanism that is more akin to that found in invertebrates. For example, in both flies and worms, the sex determination mechanism is cell autonomous and is based on the ratio between sex chromosomes and sets of autosomes (the X:A ratio) (Cline and Meyer 1996; Zarkower 2006). In Drosophila, an X:A ratio of 1 results in female (and ovary) differentiation while an $\mathrm{X}$ :A ratio of 0.5 generates males (and testes). In birds, it has not yet been established whether the sex (gonadal)-determination mechanism is dependent on a W-chromosome element or a Z-chromosome element (or both!) (Clinton 1998; Smith 2007). A $\mathrm{W}$-chromosome ovary determining mechanism is simpler in principle and would be analogous to the Sry gene: each is sex specific and only requires to be 'turned on' at the appropriate time. A Z-chromosome gene on the other hand would be present in both sexes and would either require an upstream sex-specific signal (which would then be the gonad-determining factor) or would need to function differently in both sexes.

\section{DMRT1}

Although no obvious W-chromosome candidate ovary-determining genes have been identified, a gene known as DMRT1 (doublesex and Mab-3-related transcription factor 1) is a likely Z-chromosome testis-determining gene (Raymond et al. 1999; Ferguson-Smith 2007; Nanda et al. 2008; Smith 2010). In chickens, DMRT1 is expressed at higher levels in male (ZZ) gonads than in female (ZW) gonads, both at and after the suspected point of gonadal differentiation (Smith et al. 1999). Most significantly, DMRT1 expression is elevated in embryonic female gonads 'masculinised' by the aromatase inhibitor, fadrozole (Smith et al. 2003), and experimentally reducing levels of the 
DMRT1transcript in male chick embryos resulted in, at least partial, feminisation of the left gonad into an ovary-like structure (Smith et al. 2009). In the latter study, expression of the male-specific marker, SOX9, was significantly reduced, and expression of the female marker, aromatase, was ectopically activated in male gonads. These findings suggested that the DMRT1 gene product is required for testis development in the chicken and that the effect is dose dependent. The presence of DMRT1 on the ratite $\mathrm{Z}$ chromosome and its absence from the near homomorphic W chromosome (Shetty et al. 2002) supports the suggestion that DMRT1 is required for testis development in birds.

The mechanism of action of DMRT1 in avian gonadal differentiation is unclear, although a theoretical threshold is often proposed: levels above this threshold produce testes while levels below the threshold produce ovaries. It seems unlikely that this theoretical threshold is represented by an absolute level of DMRT1 - with the variation in gene expression seen between different individual birds, one might expect high $\mathrm{ZW}$ expressers to exceed the threshold and low ZZ expressers to fail to achieve the required level. It seems more likely that the threshold is defined by the interaction of DMRT1 and some autosomal factor along the lines of the $\mathrm{X}$ :A ratio system seen in flies and worms. Under this scenario, the product of two copies of DMRT1 in males would initiate testis differentiation while the product of the single copy in females would not initiate testis differentiation.

\section{The W chromosome?}

While it seems clear that DMRT1 is required for testis differentiation, it may well be that ovary differentiation is not simply due to the lack of DMRT1 but is a process that requires the presence of a $\mathrm{W}$ chromosome. In support of this concept, a triploid line of birds produce $3 \mathrm{~A}: \mathrm{ZZW}$ hatchlings that appear phenotypically female and are described as intersex with a right testis and a left ovo-testis (Lin et al. 1995). Although the ovarian component of the left gonad is gradually lost as these birds mature, these observations suggest that the $\mathrm{W}$ chromosome may influence normal ovarian development. In this context, it is interesting to note that no avian aneuploids such as 2A:Z0 (which would be extremely informative), have
Fig. 8 Schematic illustrating CASI in male and female birds. At fertilisation, expression of sex chromosome genes imposes either a male (ZZ) or female (ZW) cell autonomous sex identity on chicken embryos. CASI is a major factor in maintaining sexually dimorphic aspects of all tissues. Gonadal hormones influence the development of reproductive tissue and affect some features of sexual appendages. Gonadal hormones have little influence on non-reproductive tissues and CASI appear to be responsible for sexually dimorphic size differences. While CASI is clearly involved in establishing and maintaining the tissue phenotype, it is not clear that CASI plays a role in triggering gonadal differentiation
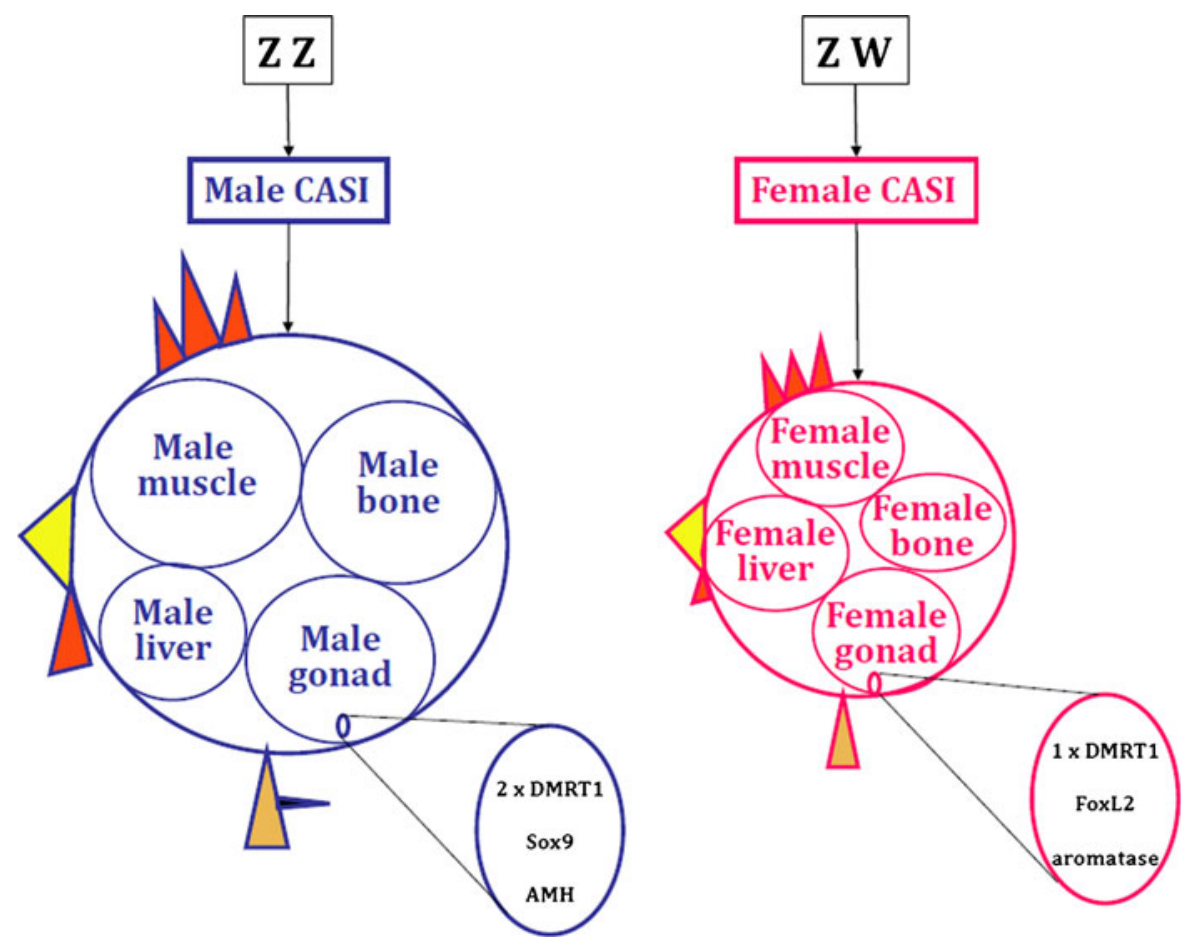
ever been identified, and it has been suggested that such genotypes may be lethal (Marshall Graves 2003).

It has been suggested that W-chromosome gene products may promote ovarian development by regulating the methylation status and expression of the MHM gene (male hypermethylation) on the $\mathrm{Z}$ chromosome, which in turn effects expression of DMRT1 (Teranishi et al. 2001; Yang et al. 2011).

\section{CASI and sex determination}

Of course, it is possible that gonadal differentiation in birds does not simply depend on sex-specific expression differences at a single locus but rather on sexually dimorphic expression at multiple loci resulting from the different sex chromosome constitutions. However, regardless of which basic mechanism may be involved, the relationship, if any, between CASI and the genes regulating gonadal differentiation is unclear. DMRT1 is a Z-chromosome gene that does not appear to be dosage-compensated, but it is not a CASI gene because it is not expressed prior to the initiation of gonadal differentiation, and DMRT1 expression is restricted to a single tissue. While it is possible that male and female CASI regulates DMRT1 expression, this seems unlikely and would require some indirect mechanism as transcription of DMRT1 occurs in both sexes, and the differences in levels of DMRT1 product seem to simply reflect the difference in gene copy number. It seems more likely that transcription of the DMRT1 gene is directly regulated by some common autosomal factor.

Although it is not clear if CASI plays a direct role in initiating gonadal differentiation, it is clear from gynandromorph studies that at some level, CASI plays a major role in determining the phenotype of all tissues (size of organs, length and density of bones, muscle mass, gonad type, etc.), and that this role is at least as significant as the role played by gonadal hormones (Fig. 8). However, it seems that under particular circumstances, the influence of CASI on the phenotype of certain tissues can be over-ridden. This is demonstrated by the fact that manipulating the gene cascade that regulates gonadal differentiation, for example, by reducing DMRT1 levels or by blocking aromatase action, will either feminise male gonads or masculinise female gonads (Elbrecht and Smith 1992; Burke and Henry 1999; Bruggeman et al. 2002; Smith et al. 2009). The resulting sex-reversed gonads will, of course, affect the development of reproductive structures and alter the appearance of hormonesensitive appendages. Interestingly, these effects are often temporary, and the gonads and other tissues can revert to the genotypic form (Burke and Henry 1999; Bruggeman et al. 2002). Most importantly, the sexual phenotype of the majority of tissues is not affected, even temporarily, by sex-reversal of the gonads. This is demonstrated by the fact that even in female birds that have been exposed to the masculinising influence of testes throughout embryonic development and sexual maturation, the overall size (organ size, bone structure, muscle mass) of the adult bird is that of a typical female (Burke and Henry 1999; Bruggeman et al. 2002). The failure of sex-reversal treatments to alter body mass and the tendency to revert to a form that accords with genotype suggests a resistance to change at the cellular levels and must explain the 'plasticity' often associated with avian development. These observations on CASI would also suggest that commercial efforts to generate malesized birds by sex-reversing female birds are unlikely to be successful.

\section{Conclusion}

While CASI is clearly involved in establishing and maintaining the tissue phenotype, it is not clear what role, if any, that CASI plays in initiating the development of any tissue, including the gonads.

Acknowledgements We gratefully acknowledge support from the BBSRC and DEFRA.

\section{References}

Agate RJ, Grisham W, Wade J, Mann S, Wingfield J, Schanen C, Palotie A, Arnold AP (2003) Neural, not gonadal, origin of brain sex differences in a gynandromorphic finch. Proc Natl Acad Sci USA 100:4873-4878

Arnold AP (1997) Sexual differentiation of the zebra finch song system: positive evidence, negative evidence, null hypotheses, and a paradigm shift. J Neurobiol 33:572-584

Aw S, Levin M (2008) What's left in asymmetry? Dev Dyn 237:3453-3463

Arnold AP (2003) The gender of the voice within: the neural origin of sex differences in the brain. Curr Opin Neurobiol 13:759-764 
Arnold AP, Chen X (2009) What does the "four core genotypes" mouse model tell us about sex differences in the brain and other tissues? Front Neuroendocrinol 30:1-9

Barnard CJ (1983) Animal behaviour: ecology and evolution. J. Wiley, New York

Baverstock PR, Adams M, Polkinghorne RW, Gelder M (1982) A sex-linked enzyme in birds-Z-chromosome conservation but no dosage compensation. Nature 296:763-766

Bellott DW, Skaletsky H, Pyntikova T, Mardis ER, Graves T, Kremitzki C, Brown LG, Rozen S, Warren WC, Wilson RK, Page DC (2010) Convergent evolution of chicken Z and human $\mathrm{X}$ chromosomes by expansion and gene acquisition. Nature 466:612-616

Blecher SR, Erickson RP (2007) Genetics of sexual development: a new paradigm. Am J Med Genet 143:3054-3068

Bowen ST, Hanson J (1962) A gynandromorph of the brine shrimp, Artemia salina. Genetics 47:277-280

Briganti F, Papeschi A, Mugnai T, Dessì-Fulgheri F (1999) Effect of testosterone on male traits and behaviour in juvenile pheasants. Ethol Ecol Evol 11:171-178

Burgoyne PS, Buehr M, McLaren A (1988) XY follicle cells in ovaries of XX-XY female mouse chimaeras. Development 104:683-688

Burke WH, Henry MH (1999) Gonadal development and growth of chickens and turkeys hatched from eggs injected with an aromatase inhibitor. Poult Sci 78:1019-1033

Bruggeman V, Van As P, Decuypere E (2002) Developmental endocrinology of the reproductive axis in the chicken embryo. Comp Biochem Physiol A Mol Integr Physiol 131:839-846

Chace FA, Moore GM (1959) A bicolored gynandromorph of the lobster, Homarus americanus. Biol Bull 116:226-231

Chue J, Smith CA (2011) Sex determination and sexual differentiation in the avian model. FEBS J 278:1027-1034

Cline TW, Meyer BJ (1996) Vive la difference: males vs females in flies vs worms. Annu Rev Genet 30:637-702

Clinton M (1998) Sex determination and gonadal development: a bird's eye view. J Exp Zool 281:457-465

Cock AG (1955) Half-and-half mosaics in the fowl. J Genet 53:49-80

Davies W, Wilkinson LS (2006) It is not all hormones: alternative explanations for sexual differentiation of the brain. Brain Res 1126:36-45

Elbrecht A, Smith RG (1992) Aromatase enzyme activity and sex determination in chickens. Science 255:467-470

Ellegren H, Hultin-Rosenberg L, Brunstrom B, Dencker L, Kultima K, Scholz B (2007) Faced with inequality: chicken do not have a general dosage compensation of sex-linked genes. BMC Biol 5:40

Fennell MJ, Scanes CG (1992) Inhibition of growth in chickens by testosterone, 5 alpha-dihydrotestosterone, and 19-nortestosterone. Poult Sci 71:357-366

Ferguson-Smith M (2007) The evolution of sex chromosomes and sex determination in vertebrates and the key role of DMRT1. Sex Dev 1:2-11

Gilbert SF (2006) Developmental biology, 8th edn. Sinauer Associates, Sunderland Mass

Graves JA (1996) Mammals that break the rules: genetics of marsupials and monotremes. Annu Rev Genet 30:233-260

Hamburger V, Hamilton HL (1951) A series of normal stages in the development of the chick embryo. Dev Dyn 195:231272
Hollander WF (1975) Sectorial mosaics in the domestic pigeon: 25 more years. J Hered 66:177-202

Itoh Y, Melamed E, Yang X, Kampf K, Wang S, Yehya N, Van Nas A, Replogle K, Band MR, Clayton DF, Schadt EE, Lusis AJ, Arnold AP (2007) Dosage compensation is less effective in birds than in mammals. J Biol 6:1-15

Johnson PT, Otto SV (1981) Histology of a bilateral gynandromorph of the blue crab, Callinectes sapidus rathbun (Decapoda:Portunidae). Biol Bull 161:236-245

Kashimada K, Koopman P (2010) Sry: the master switch in mammalian sex determination. Development 137:39213930

Kondo M, Nanda I, Hornung U, Asakawa S, Shimizu N, Mitani H, Schmid M, Shima A, Schartl M (2003) Absence of the candidate male sex-determining gene $\operatorname{dmrt} 1 \mathrm{~b}(\mathrm{Y})$ of medaka from other fish species. Curr Biol 13:416-420

Lee SI, Lee WK, Shin JH, Han BK, Moon S, Cho S, Park T, Kim H, Han JY (2009) Sexually dimorphic gene expression in the chick brain before gonadal differentiation. Poult Sci 88:1003-1015

Lin M, Thorne MH, Martin IC, Sheldon BL, Jones RC (1995) Development of the gonads in the triploid (ZZW and ZZZ) fowl, Gallus domesticus and comparison with normal diploid males (ZZ) and females (ZW). Reprod Fert Dev 7:1185-1197

Mank JE, Ellegren H (2009) All dosage compensation is local: gene-by-gene regulation of sex-biased expression on the chicken Z chromosome. Heredity 102:312-320

Marshall Graves JA (2003) Sex and death in birds: a model of dosage compensation that predicts lethality of sex chromosome aneuploids. Cytogenet Genome Res 101:278-282

Marshall Graves JA, Shetty S (2001) Sex from W to Z: evolution of vertebrate sex chromosomes and sex determining genes. J Exp Zool 290:449-462

Marshall Graves JA, Peichel CL (2010) Are homologies in vertebrate sex determination due to shared ancestry or to limited options? Genome Biol 11:205

Matsuda M, Shinomiya A, Kinoshita M, Suzuki A, Kobayashi T, Paul-Prasanth B, Lau EL, Hamaguchi S, Sakaizumi M, Nagahama Y (2007) DMY gene induces male development in genetically female (XX) medaka fish. Proc Natl Acad Sci USA 104:3865-3870

Melamed E, Arnold AP (2007) Regional differences in dosage compensation on the chicken $\mathrm{Z}$ chromosome. Genome Biol 8:R202

McGrew MJ, Sherman A, Ellard FM, Lillico SG, Gilhooley HJ, Kingsman AJ, Mitrophanous KA, Sang H (2004) Efficient production of germline transgenic chickens using lentiviral vectors. EMBO Rep 5:728-733

McQueen HA, McBride D, Miele G, Bird AP, Clinton M (2001) Dosage compensation in birds. Curr Biol 11:253-257

McQueen HA, Clinton M (2009) Avian sex chromosomes: dosage compensation matters. Chromosome Res 17:687-697

Morgan TH (1991) The physical basis of heredity. Lippincott, Philadelphia

Morrish BC, Sinclair AH (2002) Vertebrate sex determination: many means to an end. Reproduction 124:447-457

Nanda I, Schlegelmilch K, Haaf T, Schartl M, Schmid M (2008) Synteny conservation of the $\mathrm{Z}$ chromosome in 14 avian species (11 families) supports a role for $Z$ dosage in avian sex determination. Cytogenet Genome Res 122:150-156 
Ngun TC, Ghahramani N, Sanchez FJ, Bocklandt S, Vilain E (2011) The genetics of sex differences in brain and behavior. Front Neuroendocrinol 32:227-246

O WS, Short RV, Renfree MB, Shaw G (1988) Primary genetic control of somatic sexual differentiation in a mammal. Nature 331:716-717

Patek CE, Kerr JB, Gosden RG, Jones KW, Hardy K, Muggleton-Harris AL, Handyside AH, Whittingham DG, Hooper ML (1991) Sex chimaerism, fertility and sex determination in the mouse. Development 113:311-325

Raymond CS, Kettlewell JR, Hirsch B, Bardwell VJ, Zarkower D (1999) Expression of Dmrtl in the genital ridge of mouse and chicken embryos suggests a role in vertebrate sexual development. Dev Biol 215:208-220

Scholz B, Kultima K, Mattsson A, Axelsson J, Brunström B, Halldin K, Stigson M, Dencker L (2006) Sex-dependent gene expression in early brain development of chicken embryos. BMC Neurosci 7:12

Sekido R, Lovell-Badge R (2009) Sex determination and SRY: down to a wink and a nudge? Trends Genet 25:19-29

Shetty S, Kirby P, Zarkower D, Graves JA (2002) DMRT1 in a ratite bird: evidence for a role in sex determination and discovery of a putative regulatory element. Cytogenet Genome Res 99:245-251

Smith CA (2007) Sex determination in birds: HINTs from the W sex chromosome? Sex Dev 1:279-285

Smith CA (2010) Sex determination in birds: a review. Emu 110:364-377

Smith CA, McClive PJ, Western PS, Reed KJ, Sinclair AH (1999) Conservation of a sex-determining gene. Nature 402:601-602

Smith CA, Katz M, Sinclair AH (2003) DMRT1 is upregulated in the gonads during female-to-male sex reversal in $\mathrm{ZW}$ chicken embryos. Biol Reprod 68:560-570

Smith CA, Roeszler KN, Ohnesorg T, Cummins DM, Farlie PG, Doran TJ, Sinclair AH (2009) The avian Z-linked gene DMRT1 is required for male sex determination in the chicken. Nature 461:267-271

Spencer W (1927) A gynandromorph in Drosophila funebris. Am Nat 61:89-91

Staelens J, Rombaut D, Vercauteren I, Argue B, Benzie J, Vuylsteke M (2008) High-density linkage maps and sexlinked markers for the black tiger shrimp (Penaeus monodon). Genetics 179:917-925

Straub T, Becker PB (2007) Dosage compensation: the beginning and end of generalization. Nat Rev Genet 8:47-57

Teranishi M, Shimada Y, Hori T, Nakabayashi O, Kikuchi T, Macleod T, Pym R, Sheldon B, Solovei I, Macgregor H, Mizuno S (2001) Transcripts of the MHM region on the chicken $\mathrm{Z}$ chromosome accumulate as non-coding RNA in the nucleus of female cells adjacent to the DMRT1 locus. Chromosome Res 9:147-165

Wolpert L, Smith J, Jessell T, Lawrence P, Robert-son E, Meyerowitz E (2006) Principles of development, 3rd edn. Oxford University Press, Oxford

Yang X, Zheng J, Qu L et al (2011) Methylation status of cMHM and expression of sex-specific genes in adult sexreversed female chickens. Sex Dev 5:147-154

Zarkower D (2001) Establishing sexual dimorphism: conservation amidst diversity? Nat Rev Genet 2:175-185

Zarkower D (2006) Somatic sex determination. WormBook 10:1-12

Zhang SO, Mathur S, Hattem G, Tassy O, Pourquie O (2010) Sex-dimorphic gene expression and ineffective dosage compensation of Z-linked genes in gastrulating chicken embryos. BMC Genomics 11:13

Zhao D, McBride D, Nandi S, McQueen HA, McGrew MJ, Hocking PM, Lewis PD, Sang HM, Clinton M (2010) Somatic sex identity is cell autonomous in the chicken. Nature 464:237-242 